\title{
Experimental and Survey Studies on the Effectiveness of Dynamic Signage Systems
}

\author{
EDWIN R GALEA, HUI XIE and PETER J LAWRENCE \\ Fire Safety Engineering Group \\ The University of Greenwich \\ 30 Park Row, Greenwich, London SE10 9LS, UK
}

\begin{abstract}
Signage systems are widely used in the built environment to aid occupant wayfinding during both circulation and evacuation. Recent research conducted by the authors shows that only $38 \%$ of people 'see' conventional static emergency signage in presumed emergency situations in an unfamiliar built environment, even if the sign is located directly in front of them and their vision is unobstructed. However, most people who see the sign follow the sign. These results suggest that current emergency guidance signs are less effective as an aid to wayfinding than they potentially can be and that signs are likely to become more effective if their detectability can be improved while upholding the comprehensibility of the guidance information they provide. A novel dynamic signage design is proposed to address this issue. The effectiveness of the new sign is tested under almost identical experimental settings and conditions as in the previous experiments examining conventional, static signs. The results show that $77 \%$ of people 'see' the dynamic sign and $100 \%$ of them go on to follow the sign. In addition, a dynamic method to identify that an exit route is no longer viable is tested using an international survey to gauge understanding of the new signage concept. Survey results suggest that the purpose of the new sign can be clearly understood by over $90 \%$ of the sample.
\end{abstract}

KEYWORDS: human behaviour, egress, human factors, signage, wayfinding

\section{INTRODUCTION}

Signage systems are widely used in the built environment to aid occupant wayfinding during both circulation and evacuation. These include non-emergency signs, intended to assist in navigation under nonemergency circulation situations and emergency signs, intended to assist occupants locate an exit in emergency situations. These signs are particularly important where there is no direct visual access for the occupant to a potential target (exit) and orientation becomes difficult due to the lack of reference points $[1,2]$. The information conveyed by signage systems is intended to compensate for the complexity of an enclosure and/or where exits are not sufficiently apparent, thereby improving wayfinding efficiency.

Given the importance of providing information through signage systems to facilitate occupant wayfinding, there is a general lack of consideration regarding the effectiveness of occupant utilisation of this information [2,3]. Recently a series of experimental trials were conducted to study how occupants perceive, interpret and use the information conveyed by standard emergency signage [3]. The results show that only $38 \%$ of people 'see' conventional emergency signage in presumed emergency situations in an unfamiliar environment, even if the sign is located directly in front of them and their vision is unobstructed [3]. However, $100 \%$ of the people who see the sign follow the sign. These results suggest that current emergency guidance signs $[4,5]$ are less effective as an aid to wayfinding than they potentially can be. Thus signs are likely to be more effective if their detectability can be improved, while maintaining the comprehensibility of the guidance information they provide.

In order to address the problem of the low detectability of conventional, static signage, it is necessary to increase the sensory affordance of the sign $[3,6]$. The sensory affordance is provided by the features of the object that support people in sensing it; e.g. the green colour of the sign helps people in distinguishing it from the wall. There are a number of ways of enhancing the signs' sensory affordance, such as increasing the size of the sign, making the sign standout more from the background or introducing additional sensory stimuli. However, it is also essential that the simplicity and clarity of the information conveyed by the sign are not inadvertently decreased due to the change of signage design.

As part of the EU FP7 GETWAY project [7] a novel signage design is proposed to enhance the signage affordance while maintaining the maximum compliance with existing signage regulations and practice 
[4,5]. This design increases the detectability of the signs through the introduction of lit, flashing and running signage component (see Fig. 1) to the exiting standard signage design. The conventional static signage system is then turned into a dynamic signage system (DSS), whereas the size of the sign and the format of the signage information remain unchanged. The dynamic nature of the sign (i.e. the flashing cycle) is only activated during an emergency situation, when the alarm is tripped.

(1)

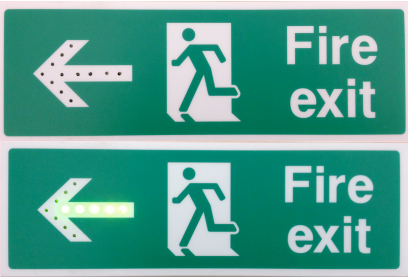

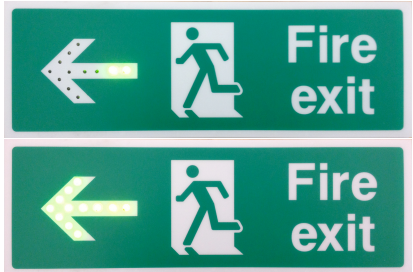

Fig. 1. The dynamic exit sign based on the standard exit sign design showing the flashing arrow in four phases.

A series of experimental trials were conducted following the same procedure as the previous trials to quantify the effectiveness of the new DSS and compare that achieved by the conventional signage system [3]. This paper presents the experimental results and the comparison with the previous trials.

In addition, as part of the GETAWAY project, the signage control system which activates the signs is intended to be intelligent. The signage control system is controlled by a Decision Engine (DE) which utilises the results from faster than real time evacuation simulations produced by a parallel implementation of the buildingEXODUS evacuation simulation software [8-10]. This enables the optimal evacuation route for the population and incident to be identified. The emergency evacuation signs that point in the appropriate exit directions are then activated by the DE. In this way, the DSS becomes an Active DSS (ADSS); and the intelligence in selecting the appropriate route and configuring the signs accordingly makes the system an Intelligent ADSS (IADSS). Furthermore, rather than simply not activating any dynamic sign pointing in what the DE considers to be hazardous directions, it was considered important to convey to the population that a normally viable exit route was no longer considered safe. Thus, exit directions which are considered potentially hazardous are effectively shut down by negating emergency exit signs pointing in those directions. As with the flashing light concept, it was desirable to develop a negated sign which could replace existing signage on a like for like basis, was easily interpreted by the population, was relatively inexpensive to install and which would fail safe should it fail to operate. Several potential concepts were considered and tested through an international survey to gauge understanding of the new signage concept. In this paper we also present the results of the international survey and demonstrate the negated sign design.

\section{THE EXPERIMENTAL METHOD}

The experiments, originally designed to evaluate the effectiveness of conventional emergency signage (discussed in detail in [3]), were replicated using the new DSS. The specific purpose of this experiment was to:

- determine the likelihood that naïve building occupants involved in an evacuation and faced with route alternatives who are located within the visual catchment area [11] of a dynamic emergency sign (i.e. have the opportunity of perceiving the sign), correctly interpret its information and correctly act upon the information;

- evaluate the level of improvement in detectability of the new dynamic sign compared with the conventional static sign;

- examine whether there is any adverse effect of the new signage design, such as causing confusion and hesitation.

The experiment was conducted at the same location using the same procedure as the previous experiment (note the glass pane in door D2 was covered in the current experiment). This represents an attempt to manage the experimental conditions and remove confounding factors such that the only key difference is the signage system being tested. The experimental trials were run in the Queen Anne Court at the 
University of Greenwich. This building was originally selected as it was readily accessible and, more importantly, the egress routes provided similar affordance in terms of lighting, configuration to limit the varieties. The building consists of staff offices, lecture halls and classrooms. The test area used was the circulation area located on the first floor of the west side of the building (see Fig. 2). This area was taken out of general use during the period of the trials to reduce the number of confounding factors that might influence participant performance.

The evacuation trials involved participants individually navigating the test area, using a route of their choice. The participants were selected from some 200 registered volunteers recruited through advertisement placed in local media and an online registration system. The selection criteria require the participants to be

- unfamiliar with the test area,

- right-handed (see results section for rationale for selecting right handed participants),

- have normal or corrected to normal vision,

- able to navigate a built environment on their own effort,

- $\quad$ aged between 18 and 70 .

Measures were also taken to ensure that an approximately equal number of male and female participants took part and that the sample was drawn from a representative distribution of ages. Finally, a total of 58 test subjects took part in the trials.

On the day of trials, participants were brought to the starting point via a route that did not include the test section. They were instructed to evacuate the building in response to the sounding of a fire alarm. The goal that they were set was to evacuate the building as quickly as possible (without running) without staff intervention or further instruction. They could select any route using their judgment unless it appeared to be unavailable (e.g. a door is locked) or if prevented by a member of staff. However, they were not specifically instructed to use the signage system. Indeed, no mention of the signage system was made during the briefing. Participants were then put through the test section individually, and their progress was recorded using a head mounted mini video camera.

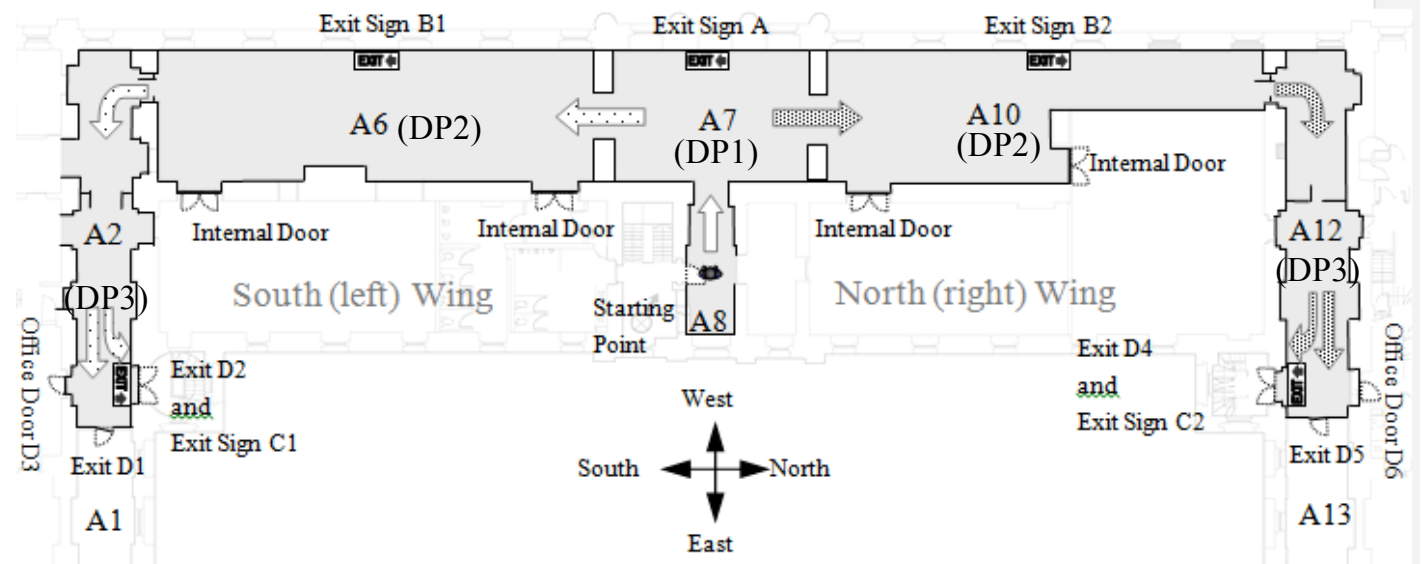

Fig. 2. The test area, exit routes, doors and location of signs used in the trials.

Each of the participants started from a short corridor (A8 in Fig. 2) running east-west. This short corridor ends in a " $\mathrm{T}$ " intersection (A7) with two adjoining corridors (A6 and A10) running south-north. The participant then chose to go left (south) or right (north) along one of the corridors. Both corridors are approximately equal in length and width, and of similar appearance. This was to limit the number of factors that might confound the results produced by artificially attracting participants to a particular route. At their widest point, both corridors are $6.8 \mathrm{~m}$ wide. The length of the south corridor (A6) is $22.9 \mathrm{~m}$ while the length of the north corridor (A10) is $22.6 \mathrm{~m}$. This junction was therefore deliberately selected given the similarity of the space size and appearance; i.e., that they afforded the evacuee equivalent alternative routes. 
On the east wall of both corridors (A6 and A10) are two interior doors (closed and locked during the trials) that lead to rooms off of the corridors, and do not present viable exits. At the south and north extreme ends of the south-north running corridors is a door leading to another corridor running east-west (A2 and A12) respectively. The east-west running corridors (A2 and A12) are approximately $2.6 \sim 3.0 \mathrm{~m}$ in width and each of these corridors runs a distance of $19 \mathrm{~m}$ and ends with three doors (all of which were closed but available during the trials): one door at the terminus of the corridor and two adjacent doors perpendicular to the direction of travel (see Fig. 2). It is considered that the participant reached the end of the trials once they passed through one of the exit points at the end of either of the corridors (A2 or A12).

During the trials, the participants travelled the same distance to reach the final exit and experienced a similar series of three decision points regardless of the exit route they used (see Fig. 2). The three decision points are:

- DP1: the "T" intersection (A7),

- DP2: the section of the south-west corridor (circulation space) leading from the "T" intersection (A6 and A10),

- DP3: the east end of each east-west corridor (A2 and A12).

At each of these decision points a dynamic exit sign was installed to highlight the appropriate path or exit; i.e. there was an opportunity for the participants to receive information from the signs at key decision points. The types of sign considered in this test were the green "running man" emergency exit signs with directional information (see Fig. 1). The signs were $0.1 \times 0.3 \mathrm{~m}$ in size, reflective in nature and were all positioned at the same height above the floor. In addition to the standard sign design, these also incorporated a lit and flashing arrow. In all cases the design of the signs complied with UK standards [4,5], except for the flashing element that was inserted over the existing static sign symbol. Given different installation locations, the signs used in the trials varied in the directional information conveyed (i.e. the direction of the arrow) and the angle at which individual participant approached the sign, depending on their location and the routes available. This was to establish the impact that the direction of approach might have upon the likelihood of seeing the sign. All the signs were located in well-lit areas illuminated by both natural and artificial lighting. In all signage installation locations, the vertical illumination measured was significantly larger than 100 lux to comply with UK standards [4].

On completion of the trial, each participant was interviewed. The interview included a questionnaire that identified the factors that assisted the participant in selecting exit paths at each of the decision points. The list of factors included familiarity, route pre-selected by participant, environmental conditions, architectural configuration and presence of signage. It should be noted that the questions did not explicitly ask participants to comment on signage but included signage within a set of multiple choice answers. Participants could also add their own comments to each question. The video footage was used later to confirm their replies concerning whether they saw the sign(s) or they missed. The video was also used to assess participants' decision times at the first decision point.

Participants' opinions towards the new DSS design were also noted if they recalled seeing the flashing component of the sign(s). Their interpretation of the DSS design was examined in the last part of the questionnaire through two questions. These two questions required the participants to indicate their level of agreement with two sets of statements based on their experiences during the trials using a five point likert scale, ranging from strongly disagree to strong agree. The first set included three statements to assess their opinions about the DSS without indicating clearly the purpose of introducing the flashing component into signage design. The three statements were:

(a) I found the flashing lights in the sign confusing.

(b) I found the flashing lights in the sign helpful.

(c) I found the flashing lights in the sign of no use. 
The second set included five statements to assess their level of agreement with statements in which the purpose of the DSS was identified i.e. assist people in making a quick route decision and reinforce this decision. The five statements were:

(a) The flashing lights in the sign assisted me in selecting which way to go.

(b) The flashing lights in the sign reinforced my decision concerning which direction to take.

(c) The flashing lights in the sign made no difference to my decision to take a particular direction or action.

(d) The flashing lights in the sign assisted me to make a quick decision as to which direction or action to take.

(e) The flashing lights in the sign made me stop and hesitate a little while deciding which direction or action to take.

It should be noted that the experimental method examines an individual's interaction with signage in ideal conditions; it does not take account of other possible influencing factors such as presence/absence of fire effluent or interaction with other occupants.

\section{THE INTERNATIONAL SURVEY METHOD}

An international web based survey (http://fseg.gre.ac.uk/WhichWay/) was undertaken to determine the most appropriate modification to the standard emergency exit sign to suggest that an exit route is no longer available. The survey involved several possible designs of negated sign (see Fig. 3) in order to determine which sign, if any, conveyed the clearest indication that the exit route originally indicated by the sign was no longer considered viable. These questions addressed the following issues: Signage Interpretation; Ranking level of agreement with the specified meaning; and Identifying which of the four options most clearly suggests that the exit route is not available.
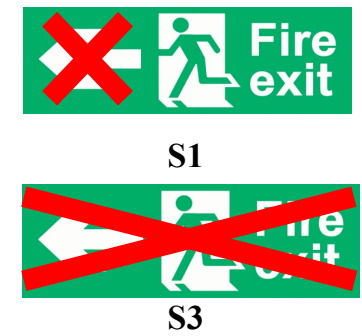

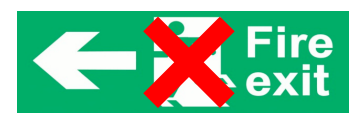

S2

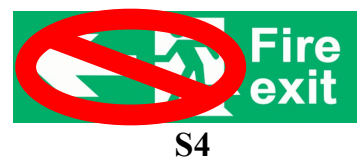

Fig. 3. Negated sign options based on the standard UK emergency exit sign.

1) Signage Interpretation: Each potential design was shown to the participant, without providing an explanation of what the sign was intended to represent. The participant was then asked to write what they thought the sign meant. The replies were classified as either correctly interpreting the meaning of the sign or incorrectly interpreting the meaning of the sign. The interpretation of each sign was independently assessed by two different assessors. The replies to the various tasks were broken down into replies from the fire professionals, non-fire professionals and overall sample. This was done to ensure that the results were not biased by the opinions of the fire professionals.

2) Ranking level of agreement: Each potential design was shown to the participant along with a sentence describing the intended meaning of the sign. The participants were then asked to rate how strongly they agreed with the provided interpretation. This was based on a five point likert scale, ranging from strongly disagree to strongly agree. The descriptive sentence that was asked for each sign was:

"The sign shown below clearly suggests to me that the emergency exit route is no longer available for use." 
3) Identifying which option most clearly suggests that the exit route is not available: All four variants were shown to the participants and they were asked to identify which one sign most clearly indicates that the exit route is no longer available.

At the time of writing, 451 people had responded to the survey from more than 10 countries. The demographics breakdown is as follows, $74 \%$ male, $25 \%$ female, with $30 \%$ in the age range $15-25 ; 46 \%$ in the age range $26-50$ and $22 \%$ in the age range $51-75$. The respondents came from the following countries; $31 \%$ from the UK; $38 \%$ from the EU (e.g. $21 \%$ Germany, $6 \%$ France, $5 \%$ Italy, $2 \%$ Netherlands); $15 \%$ from the USA and $9 \%$ from China. Of the sample, $54 \%$ described themselves as being in the fire safety profession and $45 \%$ were in other professions.

\section{EXPERIMENTAL RESULTS AND DISCUSSION}

The data obtained from the first five participants was discarded due to procedure and technical errors at the beginning of the trials. The data obtained from the other 53 participants were analysed and are presented in this section. These 53 participants included 28 males $(52.8 \%)$ and 25 females $(47.2 \%)$, and were aged between 18 and 70 .

The data presented include the number of participants who detected and utilised the DSS, the decision times (measured at the first decision point) and the participants' opinions about the DSS that they experienced. Whether or not participants detected a sign was determined primarily from the questionnaire. In addition, by examining the video recording of each participant's progress, it was often possible to determine whether a participant noted the presence of the sign and was therefore used to confirm the reported activities (e.g., from the head-mounted camera). The video footage was also used to assess the decision times at the first decision point. The participants' opinions concerning the DSS were collected from those who noticed the DSS in the trials. These participants were asked in the last part of the questionnaire about their general opinions concerning the DSS and how the DSS influenced their decisions during the trials. The analysis of the data collected is presented in the order in which the participants encountered the three decision points and the corresponding dynamic exit signs in the trials.

\section{Decision Point 1 (DP1): Exit Sign A, Route Selection at "T" Intersection A7}

The first decision point participants encountered is T-intersection A7, and the first sign encountered is sign A (see Fig.4).

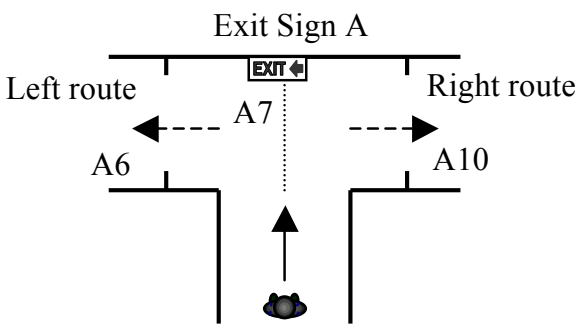

Fig.4. Participant at Decision Point 1 (T-intersection A7) is faced with Exit Sign A (pointing to the left).

The left exit route via A6 and the right exit route via A10 are similar and are therefore expected to offer the same level of affordance to the participants if additional information in the form of signage is not available (i.e. no other influencing factors are dominant in the route decision), especially given their lack of familiarity with the space. At this decision point, $12(23 \%)$ participants claimed to have not detected sign A and therefore did not use the sign provided to make a route choice. Of these, $6(50 \%)$ selected the left route and $6(50 \%)$ selected the right route. This shows the equivalent attractiveness of the options provided when the sign was not used or was not present. It should be noted that earlier research has shown that when faced with an equivalent left or right exit choice, approximately $64 \%$ of right handed people would select to go right [12]. As all of the trial participants were right handed, of the 12 participants who claimed not to have seen the sign, slightly more of the participants (7.7) may have been expected to have gone right. However, given that the sample size in this experiment is rather small - with only 12 right handed participants failing to see the sign - it is difficult to determine whether the results are in conflict with the findings from [12]. Furthermore, some of these participants may have actually seen the sign but were not 
aware that they had done so at the time and this influenced their decision to go left, resulting in the slightly higher number of people turning left than may have been expected.

Among the 53 participants, 41 (77\%) claimed that they saw an exit sign and all of them (100\%) chose to go left following the direction indicted by the sign. Thus the DSS achieves a significantly improved detection rate compared to the standard emergency sign [3]. Indeed, the detection rate for the DSS is $103 \%$ better than the conventional static signage system under the same experimental settings and conditions i.e. $77 \%$ detection rate compared with $38 \%$ detection rate. Furthermore, the difference in the detection rates achieved by the DSS and the conventional signage system is statistically significant $\left(\chi^{2}(1, N=94)=14.23\right.$, $\left.\mathrm{p}=1.62 \times 10^{-4}<0.05\right)$.

\section{Decision Point 2 (DP2): Exit Sign B1/B2, Route Selection at Corridor A6/A10}

The second decision point participants encountered is in corridor A6/A10 and the second sign encountered is sign B1/B2 (see Fig.5). The two doors located on the east wall of corridor A6/A10 (leading to rooms) complicate the wayfinding as participants may have mistaken these as being part of the exit route. Sign B1/B2, placed on the west wall opposite to the non-exit doors (with no other posters or signs in close proximity), point to the south and north end of the corridor respectively. These signs are intended to direct the participants to move towards the door at the far end of the corridor. However, unlike sign A, where participants approach perpendicular to the face of the sign (i.e. moving directly towards the sign), participants approach the B1/B2 sign at a tangent, making this sign potentially a little more difficult to be detected. In essence, there are two reasons for the increased difficulty in detection. Firstly, participants must be closer to the B1/B2 sign compared to sign A, before they can discern the information on the sign [11]. Secondly, as the trajectory of participants is at a tangent to the direct line of sight to the sign, potentially there is a smaller chance that the sign will be detected compared to the situation where participants head directly towards the sign [2].

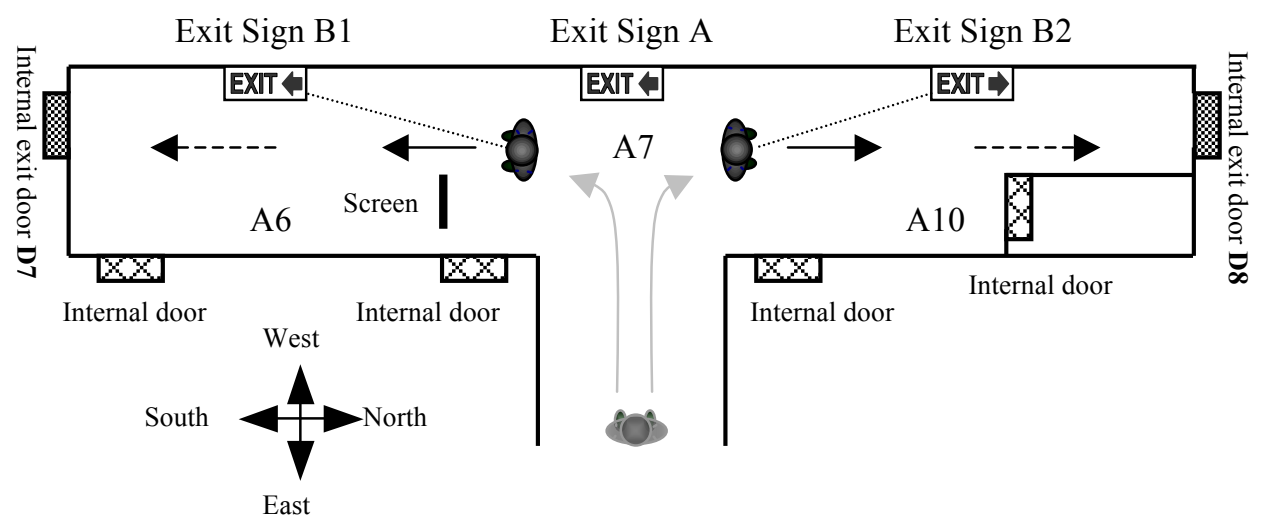

Fig.5. Decision Point 2: Exit Sign B1/B2, route selection at Corridor A6/A10.

As both sections of the south-north corridor are similar in terms of the affordance offered and the positions of sign B1/B2, the analysis of the participant behaviour in corridor A6 and A10 is therefore combined. Among the 53 participant, 38 (72\%) participants detected sign B1 or B2. Although it is potentially more difficult to detect a sign when moving parallel to the sign than when moving directly towards the sign (at a $0^{\circ}$ approach angle), the results show that the difference between the detection rates of sign $\mathrm{A}$ and sign $\mathrm{B} 1 / \mathrm{B} 2$ is not statistically significant $\left(\chi^{2}(1, \mathrm{~N}=106)=0.45, \mathrm{p}=0.50>0.05\right)$. Thus, even when approaching the dynamic sign at a tangent (parallel to the face of the sign), there is still a very high detection rate (72\%). This is significantly different to the earlier trial using static signs in which only $37 \%$ of the participants detected the static sign when approaching the sign at a tangent $[3](\chi 2(1, \mathrm{~N}=94)=11.59, \mathrm{p}=6.63 \times 10-4<0.05)$. This is an important result as it suggests that the dynamic sign can achieve a significantly high detection rate for a wide range of approach angles, even when approaching the sign on a path parallel to the sign. However, it is noted that detecting the first sign may increase the chance of detecting the second sign. 
While the dynamic sign is slightly more difficult to detect when approached on a tangential path (72\% detection rate) compared to a normal path ( $77 \%$ detection rate), the relative difference between the detection rates for the dynamic sign $(6.5 \%)$ is similar to the relative difference for the static sign (5.1\%) under similar conditions (39\% and 37\% for the normal and tangential approach respectively). As with the dynamic sign, the difference between the detection rates for the normal and tangential approaches for the static sign is not statistically significant $\left(\chi^{2}(1, \mathrm{~N}=82)=0.05, \mathrm{p}=0.82>0.05\right)$.

\section{Decision Point 3 (DP3): Exit Sign C1/C2, Exit Route Selection at Corridor A2/A12}

The third decision point encountered is A2/A12 and the third sign encountered is sign C1/C2 (see Fig.6). There are three doors to choose from at the east end of A2/A12, one directly in the path of travel (i.e. at the terminus of each corridor - D1 in A2, D5 in A12) and two adjacent doors (i.e. D2 and D3 in A2, D4 and D6 in A12). The correct exit door (i.e. indicated by the DSS placed above the door) is one of the doors to the side which leads to the emergency staircases (i.e. D2 in A2, D4 in A12). While both exit routes along the south and north east-west running corridors are almost identical, there is a difference in the configuration of the final three doors. The emergency exit door D2 (indicated by sign C1) in the south corridor A2 has an opaque glass pane which, while not transparent (covered by large white card), is different to the other two doors which are of wooden construction. The emergency exit door D4 (indicated by sign C2) in the north corridor A12 and the non-emergency exit door D5 are both solid wood doors, and the third door D6 is a locked office door with transparent glass pane. The different appearance of these two emergency exit doors may have had an impact on participant exiting decision and so it was not possible to simply combine the results from these two doors with signage; i.e., we did not wish to potentially pollute the other data by mixing the data collected. This means that the data from the south and north corridors must be analysed separately.

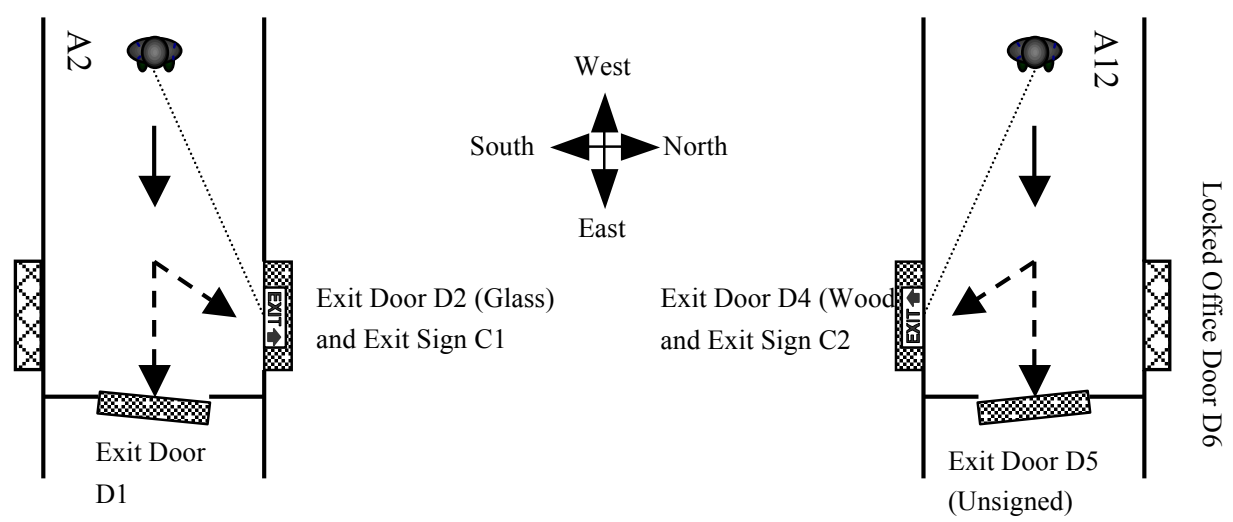

(a) Exit Sign C1, exit route selection at Corridor A2

(b) Exit Sign C2, exit route selection at Corridor A12

Fig.6. Decision Point 3 at (a) A2 and (b) A12.

\section{Exit Sign C2, Exit Selection at North Corridor A12}

There are six participants who went right from A7 and eventually reached corridor A12. As the data-set is too small to draw any firm conclusions, the results are simply presented here for completeness (see Table 1). Among the six participants, four (67\%) did not notice sign $C 2$ : two of them selected door D5 in the path of travel and the other two selected the emergency exit door D4. Among the six participants, two (33\%) claimed that they saw an exit sign at A12 and both of them selected door D4 as a result. 
Table 1: Signage detection rate and participant exit selection in A2 and A12 in current trials.

\begin{tabular}{|c|c|c|c|c|}
\hline Participants & \multicolumn{2}{|c|}{ Signage detection rate } & \multicolumn{2}{c|}{ Exit selection } \\
\hline \multirow{3}{*}{$\begin{array}{c}6 \text { participants in } \\
\text { A12 }\end{array}$} & Who did not see any sign & $4(67 \%)$ & Who chose D5 & $2(50 \%)$ \\
\cline { 4 - 5 } & & & Who chose D4 & $2(50 \%)$ \\
\hline \multirow{3}{*}{$\begin{array}{c}47 \text { participants } \\
\text { in A2 }\end{array}$} & Who saw sign C2 on D4 & $2(33 \%)$ & Who chose D5 & $0(0 \%)$ \\
\cline { 4 - 5 } & Who did not see any sign & $23(49 \%)$ & Who chose D4 & $2(100 \%)$ \\
\cline { 4 - 5 } & Who saw sign C1 on D2 & \multirow{2}{*}{$24(51 \%)$} & Who chose D1 & $18(78 \%)$ \\
\cline { 4 - 5 } & & & Who chose D2 & $5(22 \%)$ \\
\hline
\end{tabular}

\section{Exit Sign C1, Exit Selection at South Corridor A2}

There are 47 participants who went left from A7 and eventually reached corridor A2. Among the 47 participants, $23(49 \%)$ claimed that they did not notice an exit sign in relation to any door. Among them, five $(22 \%)$ chose to go through the emergency exit door D2 and the other $18(78 \%)$ chose to use the corridor door D1 in the path of travel. It is apparent that exit door D1 is considerably more attractive than exit door D2 to those who are not familiar with the building layout and did not detect the signage above the door. Note that this is different from the finding in the previous trials [3] where those who were not familiar with the building layout and did not use signage preferred exit door D2 (82\%) to D1 (18\%). This may have been because the glass pane on the top half of exit door D2 was not covered during the original trials and allowed light to come through. This gave an impression to the participants that it may provide a direct route to the exterior, hence increasing the affordance of D2. The glass pane of D2 was fully covered during the current trials. D2, as a side door, became less attractive than door D1 directly in the path of travel.

Among the 47 participants who turned left from A7, 24 (51\%) claimed that they saw sign $\mathrm{C} 1$ above the emergency exit door D2 (see Fig.6). Among them, 21 (88\%) made a decision to use exit door D2 rather than attempting to use the other exit, and $3(12 \%)$ chose exit door D1. This is consistent with the finding in the previous trials [3] where $89 \%$ of those who were not familiar with the building layout and saw sign $\mathrm{C} 1$ decided to use exit door D2. It should be noted that the detection rate achieved by the DSS in A2 is $51 \%$ (24/47), while the detection rate achieved by the conventional signage system in A2 in the previous trials [3] is 39\% (9/23). Although the DSS achieves an increase in detection rate of $31 \%$ compared with the conventional static signage, the difference in the detection rates achieved by the two signage systems is not statistically significant $\left(\chi^{2}(1, \mathrm{~N}=70)=0.88, \mathrm{p}=0.35>0.05\right)$. However, considering the relatively lower affordance of D2 in the current trials than that in the previous trials, it can be expected that the DSS should have had a more significant impact on participant exit selection than the conventional static signage in A2 (see Table 1).

\section{The influence of the DSS upon participant's decision-making time}

Exit signs are able to provide directional information to people in emergency situations and can help them make an exit decision at places where doubt may exist about the choice of escape route or exit. If people can successfully perceive and comprehend the information, it is expected that not only do they make a decision correctly, but they also act quickly. This becomes increasingly important the greater the number of decision points experienced, especially in time critical evacuation situations. This expectation was examined in the previous trials [3] in which video footage was used to estimate the participants' decisionmaking time at the first decision point; i.e. the amount of time the participants spent in determining which direction they would travel at the "T" intersection A7.

This was measured from the moment when the participant could discern that there were two possible routes to the moment when they decisively headed in a particular direction, either the left or the right (see Fig.7). 


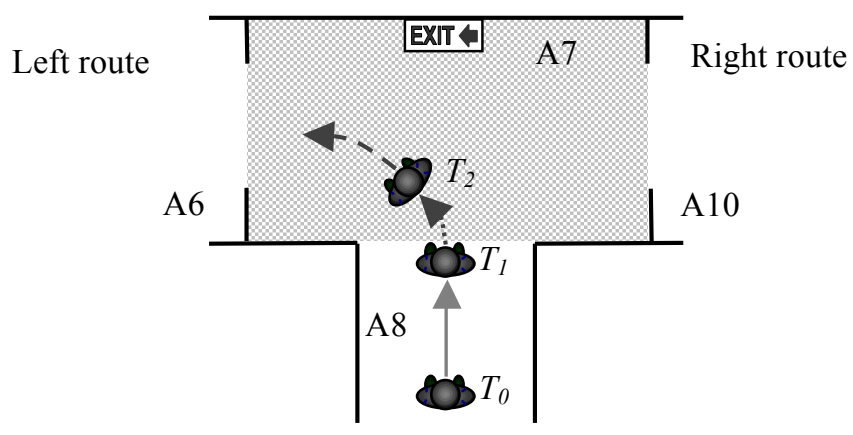

$T_{0}$ : Participant starts in corridor A8.

$T_{1}$ : Participant enters the A7 shaded decision region.

$T_{2}$ : Participant decisively heads in a particular direction.

Fig.7. Estimate participant's decision-making time at the " $T$ " intersection (decision point 1).

It was found in the previous trials using the conventional static signage that those participants who were unfamiliar with the building but who detected and used sign A had an average decision time of $2.6 \mathrm{~s}$, while those unfamiliar participants who did not detect sign A had an average decision time of $5.6 \mathrm{~s}$ [3]. The difference in decision times between those who detected the sign and those who did not is statistically significant.

The decision-making time of the 53 unfamiliar participants in the current trials using the DSS is also estimated following the same method. It is found that the 41 participants who detected sign A had an average decision time of $1.8 \mathrm{~s}$, while the 12 participants who did not use signage had an average decision time of $5.7 \mathrm{~s}$ (see Table 2 and Fig.8). The average decision time of those who did not detect the sign is $217 \%$ longer than that of those who detected the sign. The difference in the decision times between those who detected the sign and those who did not detect the sign is statistically highly significant (MannWhitney $\mathrm{U}=470.5, \mathrm{n} 1=41, \mathrm{n} 2=12, \mathrm{P}<0.001$, two-tailed).

Compared with the results obtained in the earlier trials [3], the average decision time of those who detected the conventional static sign is $44 \%$ longer than that of those who detected the new dynamic sign. However, the difference in the decision times between those who detected the static sign in the previous trials and those who detected the dynamic sign in the current trials is not statistically significant (Mann-Whitney $\mathrm{U}=267.0, \mathrm{n} 1=41, \mathrm{n} 2=11, \mathrm{P}=0.35>0.05$, two-tailed). The average decision times of those who did not detect any sign in both sets of trials are similar, as expected. The difference in the decision times between those who did not detect any sign in both sets of trials is not statistically significant (Mann-Whitney $U=145.5$, $\mathrm{n} 1=12, \mathrm{n} 2=24, \mathrm{P}=0.96>0.05$, two-tailed).

Table 2: Participant decision-making time at the " $T$ " intersection.

\begin{tabular}{|c|c|c|c|c|c|}
\hline \multirow{2}{*}{$\begin{array}{c}\text { Detected } \\
\text { Sign? }\end{array}$} & \multirow{2}{*}{$\begin{array}{c}\text { Number of } \\
\text { participants }\end{array}$} & \multicolumn{4}{|c|}{ Decision time (s) } \\
\cline { 3 - 6 } & & Minimum & Maximum & Average & STDEV \\
\hline Yes & 41 & 0.6 & 5.4 & 1.8 & 1.1 \\
\hline No & 12 & 2.2 & 12.1 & 5.7 & 2.7 \\
\hline
\end{tabular}




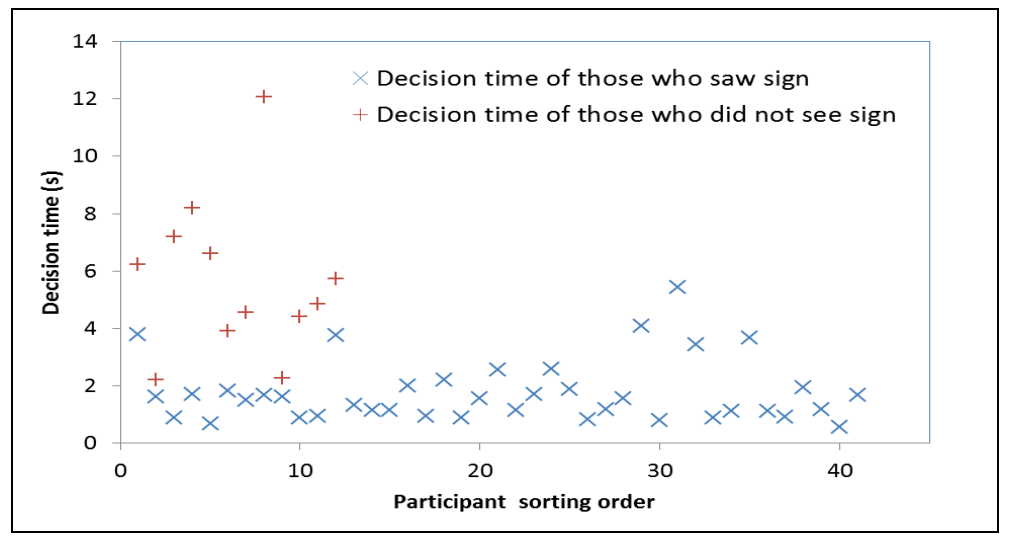

Fig.8. Participant decision-making time at the " $T$ " intersection (decision point 1).

\section{Interpretation of the dynamic signage design}

Among the total 53 participants, $46(87 \%)$ claimed that they saw one or more signs (including some fire action signs) during the trials. Of these, $44(83 \%)$ claimed that they saw at least one of the exit signs during the trials. Among them, five $(11 \%)$ could not recall any flashing component on the exit sign(s) they noticed. The analysis of the interpretation of the DSS design is based on the $39(89 \%)$ participants who can confirm seeing the flashing component on the exit sign(s) they noticed during the trials.

The results for statement set 1 can be summarized as follows:

- $\quad \mathbf{9 4 . 9 \%}$ disagree $(71.8 \%$ strongly disagree and $23.1 \%$ disagree) and $\mathbf{0 . 0 \%}$ agree that "the flashing lights are confusing".

- $\quad \mathbf{8 9 . 7 \%}$ agree (56.4\% strongly agree and $33.3 \%$ agree) and $\mathbf{2 . 6 \%}$ disagree that "the flashing lights are helpful'.

- $\quad \mathbf{8 7 . 2 \%}$ disagree (46.2\% strongly disagree and $41.0 \%$ disagree) and $\mathbf{2 . 6 \%}$ agree that "the flashing lights are of no use".

The results for statement set 2 can be summarized as follows:

- $\quad \mathbf{8 4 . 6 \%}$ agree (43.6\% strongly agree and $41.0 \%$ agree) and $7.7 \%$ disagree that "the flashing lights assisted them in selecting which way to go".

- $\quad \mathbf{8 7 . 2 \%}$ agree $(48.7 \%$ strongly agree and $38.5 \%$ agree) and $\mathbf{1 0 . 3 \%}$ disagree that "the flashing lights reinforced their decision concerning which direction to take".

- $\quad \mathbf{8 1 . 6 \%}$ disagree (55.3\% strongly disagree and $26.3 \%$ disagree) and $\mathbf{1 0 . 5 \%}$ agree that "the flashing lights in the sign made no difference to my decision to take a particular direction or action".

- $\quad \mathbf{8 2 . 1 \%}$ agree (59.0\% strongly agree and $23.1 \%$ agree) and $\mathbf{1 0 . 3 \%}$ disagree that "the flashing lights in the sign assisted me to make a quick decision as to which direction or action to take".

- $\quad$ 87.2\% disagree (38.5\% strongly disagree and $48.7 \%$ disagree) and $\mathbf{1 0 . 3 \%}$ agree that "the flashing lights in the sign made me stop and hesitate a little while deciding which direction or action to take".

Thus the high signage detection and compliance rates observed in the experiments are supported by the result of the post-trial interviews. A significant majority of the participants (about $80 \%$ to $90 \%$ ) confirmed that the flashing lights in the signs assisted them in making a quick route decision and reinforced this decision. Furthermore, the first statement of question 1 and the fifth statement of question 2 attempts to examine whether there is any adverse effect of the DSS, such as causing confusion and hesitation. The answers to these questions suggest (87.2\%) that the flashing lights did not cause confusion and hesitation to the participants. In each of the questions asked, the data indicates that the presence of the DSS was perceived to be positive by the vast majority of the participants. 


\section{RESULTS FROM THE INTERNATIONAL SURVEY CONCERNING NEGATED SIGN DESIGN}

1) Signage Interpretation: The results from the signage interpretation question are presented in Table 3 . It is noted that not all the 451 participants answered all the questions. Some participants were also excluded as they provided what were considered to be farcical responses.

Table 3: Participants who correctly interpreted the meaning of the sign.

\begin{tabular}{|r|c|c|c|}
\hline & Non-Fire Correct & Fire Correct & Total Correct \\
\hline & $93 \%(193$ total $)$ & $93 \%(238$ total $)$ & $93 \%(431$ total $)$ \\
\hline & $85 \%(196$ total $)$ & $83 \%(240$ total $)$ & $84 \%(436$ total $)$ \\
\hline & $72 \%(182$ total $)$ & $79 \%(227$ total $)$ & $76 \%(409$ total $)$ \\
\hline
\end{tabular}

Clearly, the sign which has the highest level of understanding is the sign with the large red cross (S3), with $93 \%$ of the sample providing the correct interpretation. This compares with only $59 \%$ correct interpretation for the poorest performing sign (red cross over the running man - S2). It is intereseting to note that the symbol generally taken to mean negation (circle with the slash - S4) provided the second worst correct interpretation $(76 \%)$. It is also informative to view some of the incorrect interpretations for each sign. For the sign with the red cross over the arrow (S1), a common response was, go through the door, but don't go in the direction of the arrow or fire exit is here and not to the left. For the sign with the red cross over the running man (S2), many people suggested that this meant that you should not run to the exit. Others suggested that this meant that the exit was not appropriate for people. For the sign with the circle with slash (S4), a common response was that the participant was confused and didn't know what the sign meant. Finally, the sign with the large red cross through the entire sign (S3) was correctly interpreted by most people; however, some suggested that it could mean that the sign is no longer in use.

2) Ranking level of agreement: The results from the ranking of the level of agreement with the statement can be summarized as follows:

- S1: $\mathbf{5 4 . 6 \%}$ agree (17.1\% strongly agree and $37.5 \%$ agree) and $\mathbf{3 6 . 2 \%}$ disagree (13.8\% strongly disagree and $22.4 \%$ disagree).

- S2: $42.9 \%$ agree (14.2\% strongly agree and $28.7 \%$ agree) and $41.3 \%$ disagree $(14.6 \%$ strongly disagree and $26.7 \%$ disagree).

- S3: $\mathbf{8 3 . 5 \%}$ agree (55.0\% strongly agree and $28.6 \%$ agree) and $\mathbf{9 . 9 \%}$ disagree $(6.1 \%$ strongly disagree and $3.8 \%$ disagree $)$.

- S4: $44.9 \%$ agree (14.4\% strongly agree and $30.5 \%$ agree) and $\mathbf{3 8 . 2 \%}$ disagree $(12.6 \%$ strongly disagree and $25.6 \%$ disagree).

The sign with the large red cross through the entire sign (S3) had by far both the greatest level of agreement with the statement and the smallest level of disagreement with the statement. The S3 sign had almost twice the level of agreement as the other signs. 
3) Identifying which option most clearly suggests that the exit route is not available: The overwhelming majority of the participants (84\%) selected sign S3 (see Table 4).

Table 4: Participants who identified which sign best represented the concept that the exit route was no longer viable.

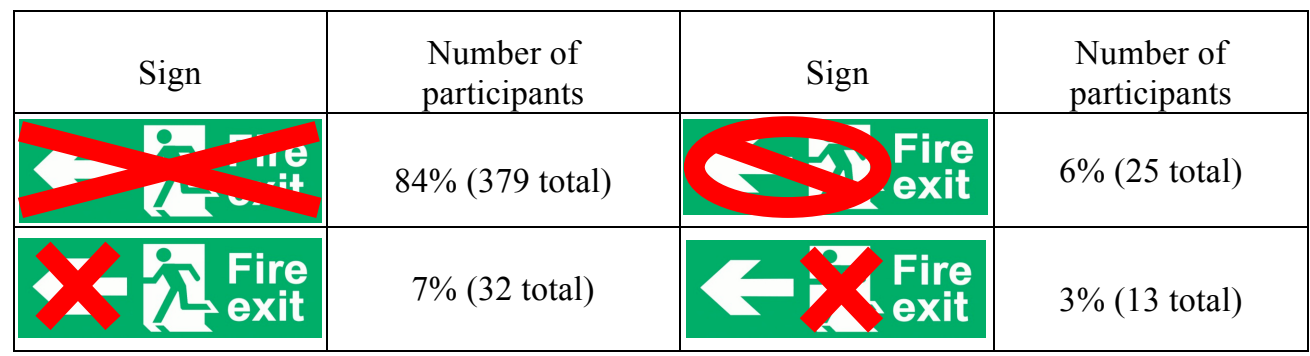

Clearly, the negated sign design that has the best interpretation and recognition is the sign with the large red cross through the entire sign (S3). This negated sign design was therefore adopted by the GETAWAY project. A prototype dynamic sign that incorporates both the flashing arrow and the large red cross has been developed for further testing in the GETAWAY project (see Fig. 9). If the exit route indicated by the sign is considered to be non-viable, the red arrow is activated. The majority of red diodes in the cross are static and, once the sign is activated remain on throughout the evacuation. However, the first and last two diodes in the cross on the left and right side flash alternatively, drawing attention to the sign. This concept will be tested in a full-scale evacuation experiment to be conducted in a rail station in Barcelona in June 2013 as part of the GETWAY project.

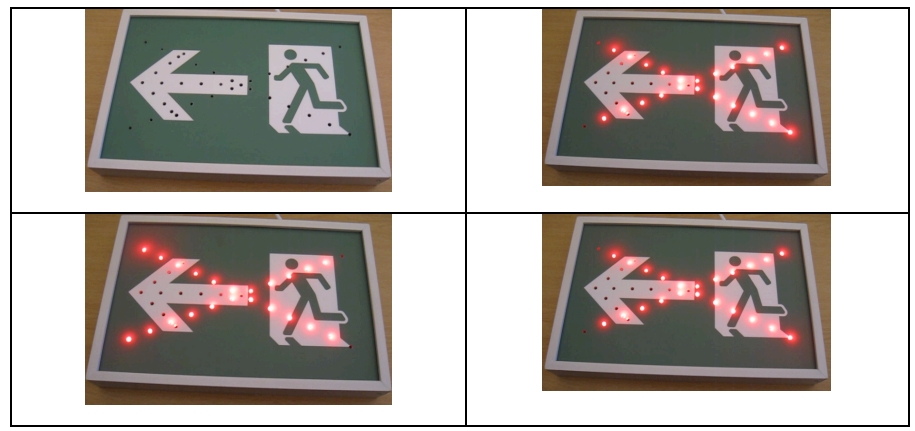

Fig.9. Proposed negated sign in operation depicting a single operation cycle.

\section{CONCLUSIONS}

Previous research has shown that only $38 \%$ of people 'see' conventional static emergency signage in presumed emergency situations in an unfamiliar built environment, even if the sign is located directly in front of them and their vision is unobstructed. However, $100 \%$ of people who see the sign follow the instructions provided by the sign. These results suggest that current emergency guidance signs are less effective as an aid to wayfinding than they potentially can be, given that they are not always noticed by those who might make use of them. This deficiency is addressed through the design and development of a new type of Dynamic Signage System (DSS), which incorporates lit, flashing and running signage component into the current standard signage design. In order to quantify the effectiveness of the new DSS a series of trials were conducted following the same procedure as the earlier experimental trials allowing direct comparison to be made.

These trials have demonstrated that the DSS achieves a detection rate of $77 \%$ when participants directly approached the sign (i.e. approach angle perpendicular to the surface of the sign at DP1) and $72 \%$ when participants approached at an angle (i.e. approach path tangent to the surface of the sign at DP2). Under the same experimental conditions, static signs produced detection rates of $39 \%$ and $37 \%$ respectively. This is an important result as it suggests that the dynamic sign can achieve a significantly high detection rate for a 
wide range of approach angles. The difference in the detection rates achieved by the DSS compared with the conventional static signage system is statistically significant. As most of the participants chose the direction indicted by the sign upon detecting the sign in both sets of trials, the increase in detection rate means the DSS had a more significant impact on participant exit selection than the conventional static signage. A similar trend was also observed for the other dynamic signs positioned in the locations where there were other influencing factors such as the presents of additional doors (Note that at the two DP3s, the data collected at North Corridor A12 is insufficient to draw any firm conclusion; however, the data collected at South Corridor A2 suggests that the DSS should have had a more significant impact on participant exit selection than the conventional static signage too). The high signage detection and compliance rates were also supported by the result of the post-trial interviews. A significant majority of the participants (about $80 \%$ to $90 \%$ ) confirmed that the flashing lights in the signs assisted them in making a quick route decision and reinforced this decision, while the flashing lights did not cause confusion and hesitation to them.

The DSS also helped people to reduce their decision times. The 41 (out of 53) participants who detected the sign required $1.8 \mathrm{~s}$ on average to decide on a route (i.e. to follow the sign), while the other 12 participants who did not see the sign spent on average $5.7 \mathrm{~s}$ in deciding upon a route. The difference in the decision times between those who detected the DSS and those who did not is statistically highly significant. Furthermore, the average detection time for standard emergency signs is $44 \%$ longer than that of those who detected the DSS, although the difference is not statistically significant.

These results suggest that the dynamic nature of the proposed emergency exit sign greatly enhances the effectiveness of emergency exit signs, making them significantly more likely to be detected. As a result, it is suggested that by using the DSS more people will be able to identify the correct exit route during an emergency evacuation than would be expected to do so using a conventional emergency signage system, thereby making the evacuation more efficient and safer. In addition, several new design concepts to indicate that an exit route highlighted by an emergency sign is no longer viable were tested via international survey. The results demonstrate that a simple red cross passing through the entire sign is correctly interpreted by over $90 \%$ of the sample as indicating that the exit route is no longer considered viable and so should not be used. Thus in addition to highlighting the desirable emergency exit route, dynamic signs may also be able to highlight which routes should not be taken. These aspects of the dynamic signage system will be tested in full-scale evacuation experiments in a railway station as part of the GETAWAY project.

\section{ACKNOWLEDGEMENT}

Project GETAWAY (contract 265717) is funded under the European Union Framework 7 Transport initiative. The authors acknowledge the co-operation of their project partners: BMT Belgium, Vision Semantics Ltd UK, London Underground Ltd UK, Evaclite Ltd UK, Hochiki Europe UK, Kingfell Bulgaria and FGC Spain; in undertaking this work and in allowing the project findings to be published.

\section{REFERENCES}

[1] Arthur, P., Passini R., Wayfinding: people, signs and architecture, pp76-81, ISBN 0-07-551016-2, 1992.

[2] Filippidis, L., Galea, E.R., Gwynne, S., Lawrence, P.J., 2006. Representing the Influence of Signage on Evacuation Behaviour within an Evacuation Model. Journal of Fire Protection Engineering, 16(1), pp.37-73, http://dx.doi.org/10.1177/1042391506054298.

[3] Xie, H., Filippidis, L., Galea, E.R., Blackshields, D., Lawrence, P.J., 2012. Experimental analysis of the effectiveness of emergency signage and its implementation in evacuation simulation, Fire and Materials; 36:367-382.

[4] British Standards Institution, 2000. BS 5499-4:2000 Safety signs, including fire safety signs Part4: Code of practice for escape route signing, BSI: London, ISBN 0580332055.

[5] British Standards Institution, 2011. BS ISO 3864-1:2011 Graphical symbols. Safety colours and safety signs. Design principles for safety signs and safety markings, BSI: London, ISBN 9780 580665158 . 
[6] Nilsson, D., Frantzich, H., Saunders, W., "Influencing Exit Choice in the Event of a Fire Evacuation.”, Fire Safety Science - Proceedings of the ninth Symposium, IAFSS, 2008, pp 341352. http://dx.doi.org/10.3801/IAFSS.FSS.9-341

[7] GETAWAY (Generating simulations to Enable Testing of Alternative routes to improve WAYfinding in evacuation of overground and underground terminals), project grant agreement, THEME SST.2010.4.1-1, EU FP7, grant agreement 265717, 07/07/11.

[8] Galea, E.R. Lawrence, P.J. Filippidis L., Blackshields, D.and Cooney, D., buildingEXODUS version 4.0: User Guide and Technical Manual, University of Greenwich, 2006.

[9] Grandison A; Muthu Y; Lawrence P; Galea E, Simulating the Evacuation of Very Large Populations in Large Domains using a Parallel Implementation of the buildingEXODUS Evacuation Model.", Proc of the 11th Int Fire Science \& Engineering Conference, Interflam, Royal Holloway College, UK, Volume 1, pp. 259-270. ISBN 9780 9541216-8-6, 2007.

[10] Galea E. R., Wang Z., Veeraswamy A., Jia F., Lawrence P. J. and Ewer J., “Coupled fire/evacuation analysis of station nightclub fire", Fire Safety Science -- Proceedings of the Ninth International Symposium, International Association for Fire Safety Science, 2008, Karlsruhe, Germany, pp 465-476. http://dx.doi.org/10.3801/IAFSS.FSS.9-465

[11] Xie, H., Filippidis, L., Gwynne, S., Galea E.R., Blackshields, D., Lawrence, P.J., 2007. Signage legibility distances as a function of observation angle, Journal of Fire Protection Engineering, 17(1), pp.41-64.

[12] Veeraswamy, A., Galea, E.R., Lawrence, P.J., 2011. Wayfinding Behaviour within Buildings An International Survey, Proceedings of the Tenth International Symposium on Fire Safety Science, University of Maryland, 19-24 June 2011, pp. 735-748. ISSN 1817-4299. http://dx.doi.org/10.3801/IAFFS.FSS.10-735. 